\title{
Riconsiderazioni sull'Anonimo Londinese: progressi e punti ancora irrisolti
}

\author{
Daniela Manetti
}

Se l'edizione principe di Hermann Diels del papiro noto come "l'Anonimo Londinese" (P.Lond. inv. 137 = P.Lond.Lit. 165) dal $1893^{1}$ è restata un punto di riferimento degli studi fino agli anni ottanta del ' 900 , a partire dalla seconda edizione critica del 2011 a cura di Daniela Manetti (Teubner, Berlin), in poco tempo ha preso slancio un grande rinnovamento di ricerche, che ha visto venire alla luce una terza edizione, con traduzione francese, a cura di Antonio Ricciardetto, prima pubblicata nella serie "Papyrologica Leodiensia" 4 (Liège 2014) e poi nella prestigiosa "Collection des Universités de France" (Paris 2016). Il papiro londinese, databile verso la fine del I sec. d.C., che risulta essere il rotolo conservato più lungo di carattere medico $(335 \mathrm{~cm})$, contiene un testo abbastanza complesso, con una prima parte dedicata alla definizione dei concetti base di 'affezione' e malattia del corpo e dell'anima (i 1 - iv 17), seguita da una sezione dossografica sulla eziologia delle malattie (iv 18 - xxi 8), e poi da una sezione dedicata alla fisiologia umana, in particolare alla digestione dei cibi (xxi 9 - xxxix 32). Oggi è opinione comune che il papiro sia un esempio di scritto autografo ${ }^{2}$.

Antonio Ricciardetto aveva rivolto l'attenzione ad un aspetto particolare di P.Lond. inv. 137 già in un articolo dedicato alla Lettera di Marco Antonio al Koinon di Asia, che è trascritta (da altra mano) sul verso ${ }^{3}$, ma in questi ultimi anni ha sviluppato ulteriori approfondimenti sugli aspetti bibliologici del rotolo e sui suoi segni di punteggiatura ${ }^{4}$. Ricciardetto ha il merito di avere fatto buoni progressi con la sua edizione nella trascrizione papirologica del testo ${ }^{5}$ e in particolare dei segni, identificando anche una coronide che non era stata riconosciuta e dando rilievo all'uso (una sola volta) di un tratto obliquo ${ }^{6}$. Inoltre

\footnotetext{
${ }^{1}$ Diels, Anonymi.

2 L'ipotesi, formulata da D. Manetti già nel 1986, fu esposta in dettaglio con dovizia di documentazione in Manetti, Autografi, ed è stata accettata da altri studiosi, da ultimo Ricciardetto: per una sintesi vedi Ricciardetto, Anonyme, XLI-XLIII.

${ }^{3}$ Ricciardetto, Lettre.

${ }^{4}$ Ricciardetto, Comparaison; poi Id., Spazio scritto e Abréviations.

${ }^{5}$ Per un elenco di correzioni di imprecisioni ed errori di Manetti, Anonymi, vd. Ricciardetto, Recensione.

${ }^{6}$ Ricciardetto, Anonyme, XXIV-XXXV; Id., Abréviations.
} 
ha collocato il sistema delle numerose abbreviazioni e dei segni di lettura nel contesto di papiri affini per situazione e datazione come quello (P.Lond. I 131v = P.Lond.Lit. 108) dell'Athenaion Politeia di Aristotele, che fa parte dello stesso lotto di papiri nel quale anche l'Anonimo giunse al British Museum.

Il che ci porta al problema del contesto di ritrovamento dell'Anonimo Londinese: in effetti il gruppo di papiri che furono acquistati e dal 1889 pervennero a Londra (si tratta dei P.Lond. inv. 130-137, con l'aggiunta di una scatola di frammentini nel 1900), è del tutto eccezionale, perché comprende una serie di rotoli letterari di grandissima importanza, primi fra tutti quelli (4) che ci hanno fatto recuperare l'Athenaion Politeia di Aristotele, ma che contengono anche l'inizio di un commento a In Midiam di Demostene e note da un commento a Callimaco; un rotolo che conserva la maggior parte di Isocrate De pace, e poi un altro contenente Demostene, Epistula III e Iperide In Philippidem; uno che ci ha fatto conoscere i Mimi di Eroda, un altro che conserva frammenti di Omero Iliade III e IV libro. Fra di essi l'Anon. Lond. (inv. 137) risulta essere l'unico testo medico. Oggi si ritiene che, al di là delle notizie confuse e forse volutamente depistanti delle memorie dei personaggi coinvolti nelle scoperte di quegli anni (E. A. W. Wallis Budge e A. H. Sayce, e in ruolo di intermediario J. Alexander) e dei resoconti amministrativi ufficiali del British Museum, il luogo del ritrovamento non fosse il nominato villaggio di Meir, ma più probabilmente la capitale del nomo, Hermopolis e che il luogo non fosse una tomba, bensì un' abitazione. L'analisi critica delle fonti fu iniziata da Guido Bastianini in un articolo del $1996^{7}$ e ha avuto poi un certo sviluppo, fino al contributo di Demokritos Kaltsas del 2015, alla cui sintesi rimando ${ }^{8}$.

La possibilità di considerare questi manoscritti come facenti parte di un 'gruppo' unitario (ipotesi che viene ammessa come probabile anche da Kaltsas) fu suggerita per la prima volta nel 2003 da Gabriella Messeri ${ }^{9}$, che riconobbe nei manoscritti molti elementi comuni come la scrittura informale (con molte abbreviazioni) e la copia personale di testi sul verso di rotoli usati. In seguito, Lucio Del Corso ha analizzato nel 2008 proprio la possibilità che questo gruppo di testi identificasse un "gruppo di scrittura", cioè un gruppo di persone con la stessa educazione di buon livello, gli stessi interessi culturali e disponibili a copiare personalmente testi di loro interesse ${ }^{10}$. Che questo permetta di inquadrare testi profondamente diversi fra di loro in uno stesso orientamento, chiamiamolo di studio, è certamente ipotesi suggestiva, ma resta tuttavia un'ipotesi, vista l'incertezza delle fonti (resta sempre la possibilità che i venditori locali abbiano volutamente suggerito l'ipotesi di un ritrovamento unico per rendere più interessante l'offerta). Inoltre Del Corso si spinge a dare un

\footnotetext{
${ }^{7}$ Bastianini, Luogo.

${ }^{8}$ Kaltsas, Randnotizen: a p. 259 egli mette in luce altri elementi di carattere documentario a favore di Hermopolis.

${ }^{9}$ Messeri, PLitLond 131, 23-6.

${ }^{10}$ Del Corso, Politeia.
} 
profilo dei possessori / fruitori:

Tutto lascia pensare, insomma, che la 'biblioteca della Politeia' - indipendentemente dall'identità del possessore dei volumina e del luogo fisico in cui essi erano conservati al momento del loro rinvenimento moderno - fosse a disposizione di un gruppo animato da comuni interessi intellettuali (come la passione per la letteratura e in particolare per la retorica): una sorta di synedrion - nel linguaggio teofrasteo una vera e propria cerchia di amici che hanno compiuto insieme gli studi - composto non tanto (e comunque non solo) da intellettuali professionisti (insegnanti di retorica? filosofi? medici?), quanto piuttosto da gentiluomini di provincia impegnati nell'amministrazione pubblica, da possidenti, da funzionari, da notabili locali ${ }^{11}$.

Una caratterizzazione così precisa non ha in realtà elementi oggettivi di supporto e presuppone una omogeneità eccessiva all'interno di questo gruppo di testi: almeno l'Anonimo Londinese si distacca dagli altri, perché, se è vergato come gli altri in una scrittura informale, non è copia di un testo, ma un'opera autografa; è l'unico testo di medicina (l'autore si dichiara esplicitamente medico); utilizza una grande quantità di fonti scritte per la sua composizione, il che rimanda ad una ampia disponibilità di testi di carattere specialistico. D'altra parte l'ambiente di Hermopolis, su cui tutti ormai sono d'accordo, può essere davvero definito 'provinciale'? Ricordo che da Hermopolis provengono - limitandoci ai primi due secoli dell'età imperiale - un testo di Corinna con scoli (BKT V.2, pp. 19-55, del I/II d.C. = M-P ${ }^{3} 251$ ), un commento al De Pace di Demostene (BKT IX 91 del II d.C. $=$ M-P $\mathrm{P}^{3}$ 262.010), il famoso commento di Didimo alle Filippiche di Demostene (BKT I, pp. 4-72 = P.Berol. inv. 9780 del II d.C. $=$ M-P ${ }^{3}$ 339), testi di Euripide con marginalia (BKT V.2, pp. 73-9 = P.Berol. inv. 13217, del I/II d.C. = M-P $\left.\mathrm{P}^{3} 337\right)$; l'opera di Ierocle stoico, scritta sul verso del commento di Didimo citato (BKT IV, pp. 6-47 = P.Berol. inv. 9780v = M-P P $^{3}$ 536, della fine del II d.C.), ovviamente molti papiri omerici, ma anche il commento al Teeteto di Platone (BKT II, pp. 3-51 = P.Berol. inv. $9782=$ M-P ${ }^{3}$ 1393, del II d.C.) ecc. Un contesto in cui compaiono opere di così grande erudizione è relativamente coerente con quanto è presupposto dall'opera dell'Anonimo: l'autore ha infatti a disposizione una vasta gamma di fonti scritte, che sono manuali di etica, che sono testimoniati anche in testi stoici (come l'opera di Andronico di Rodi o di Ario Didimo), ma sono confluiti anche in autori del medioplatonismo come Alcinoo o Plutarco; manuali di definizioni dei concetti base della medicina, che si sono conservati in testi attribuiti a Galeno, come le Definizioni mediche o Introduzione o il medico; una ampia dossografia 'aristotelica' sulle cause di malattie, che, se non risale ad

\footnotetext{
${ }^{11}$ Del Corso, Politeia, 48.
} 
Aristotele stesso, certamente risale al primo Peripato, ed è consultata, pare, direttamente $^{12}$; egli dimostra familiarità con testi ippocratici, ma anche di Aristotele e di Platone, al di là della fonte dossografica, che pure li menziona, e con una letteratura, di impostazione dialettica, sui principali autori della medicina ellenistica, Erofilo, Erasistrato e Asclepiade, in qualche caso certamente mediata da opere di Alessandro Filalete, capo di una scuola medica erofilea presso il santuario di Men Karou, ad Attouda in Caria, fra I a.C. e I d.C. ${ }^{13}$ Tutto questo fa pensare all'esistenza di una biblioteca ricca e specializzata: il carattere estremamente composito del linguaggio dell'Anonimo suggerisce che egli compili la sua opera attraverso una consultazione diretta di diverse fonti, piuttosto che derivare da una fonte precedente a sua volta compilatoria ${ }^{14}$. Questo naturalmente riguarda il momento della scrittura del testo, cioè l'inizio della vita del rotolo. Dobbiamo infatti essere consapevoli che, fra inizio e fine della vita di un rotolo, molte cose di cui non sapremo mai nulla possono accadere e il manoscritto può finire abbandonato/conservato in un posto differente da quello della sua origine: è anche possibile che il papiro abbia solo finito la sua vita a Hermopolis e allora il contesto di origine sarà più chiaro una volta che si sia approfondito lo spessore culturale e la destinazione di questo testo.

In effetti l'Anonimo Londinese presenta un'altra caratteristica che lo differenzia dagli altri, cioè la presenza sul verso di un testo (per quanto scritto da altra mano più o meno coeva) che rimanda a un contesto storico e geografico lontano dall'Egitto del I sec. d.C., cioè la Lettera di Marco Antonio al Koinon d'Asia: essa risale al 42/41 o al 33/32 a.C e conferma ed amplia una serie di privilegi, su sollecitazione del suo amico e allenatore ( $\dot{\alpha} \lambda \varepsilon \dot{\varepsilon} \pi \tau \eta \varsigma)$ Marco Antonio Artemidoro avvenuta ad Efeso, alla corporazione dei "vincitori dei giochi sacri venuti dal mondo intero e dei vincitori coronati". Concede inoltre il diritto di affiggere pubblicamente una tavoletta di bronzo con la notizia dei privilegi ${ }^{15}$. L'interpretazione di questo documento ha due aspetti, da una parte chiarire a chi Marco Antonio concede certi privilegi, dall'altra spiegare la presenza di una sua copia sul verso dell'Anonimo Londinese.

L'identificazione della corporazione oscilla negli studi fra un'associazione di artisti dionisiaci, un'associazione atletica, oppure un' associazione che comprende sia atleti sia artisti vincitori di competizioni ${ }^{16}$. La menzione dell'allenatore Marco Antonio Artemidoro è naturalmente a favore della presenza di atleti, tuttavia rimangono incertezze.

Ricciardetto ha dato una nuova interpretazione, collegando la lettera di Marco

\footnotetext{
${ }^{12}$ Manetti, Doxography.

${ }^{13}$ Str. XII 8,20: cf. Nissen, Asclépios, 197-215

${ }^{14}$ Manetti, Authorial Presence, 159-78.

${ }^{15}$ Ricciardetto, Lettre, 45 . Egli riconsidera tutta la bibliografia precedente.

${ }^{16}$ Sintesi della storia critica in Ricciardetto, Lettre, 52-3.
} 
Antonio all'esistenza a Efeso di un Mouseion, in cui un'associazione di medici organizzava delle gare in onore di Asclepio. È l'unico caso a noi noto di giochi riservati a medici e le epigrafi parlano di due giorni e di quattro prove, syntagma, cheirourgia, problema, organa. Ricciardetto identifica la corporazione della lettera nella associazione di medici che organizzava i giochi in onore di Asclepio. Ma collegare un testo del I sec. a.C., in cui non si nomina alcuna divinità e si nomina invece una problematica associazione di "vincitori dei giochi sacri e vincitori coronati", a giochi dedicati ad Asclepio attestati solo in epigrafi della metà del II sec. d.C. lascia molte perplessità ${ }^{17}$. Inoltre egli sulla base di questa prima ipotesi sostiene che l'Anonimo Londinese era interessato a partecipare ai giochi e ha composto il testo, come esercizio, in funzione della prova problema ${ }^{18}$ e per questo ha anche fatto copiare il testo sul verso. Costruisce quindi ipotesi su ipotesi, dando per scontate troppe cose, non ultima il significato delle quattro prove attestate nelle epigrafi, la cui natura resta incerta ${ }^{19}$.

Tuttavia anche le altre ipotesi formulate per spiegare la presenza di questo testo nell' Anonimo sono insoddisfacenti: Ebert ${ }^{20}$ ipotizzava che un medico in viaggio in Asia avesse copiato il testo per un suo interesse verso Antonio 'l'Egiziano'; Manetti ${ }^{21}$ insiste su una connessione geografica: l'Anonimo mostra di utilizzare l'opera di Alessandro Filalete, che fu a capo di una scuola erofilea nel santuario di Men Karou in Caria e una copia della lettera di Marco Antonio è attestata in una epigrafe (più tarda) di Tralles che si trova in un'area vicina: da qui l'ipotesi che l'autore potesse avere frequentato quell'ambiente. Ma questo è insufficiente a spiegare il motivo della copia della lettera. Lucio Del Corso ${ }^{22}$ invece spiega che la lettera, come altre lettere 'ufficiali', circolava in Egitto per ragioni scolastiche

${ }^{17}$ Ricciardetto, Anonyme, CXXX-CXXXVIII. Il fatto che alcuni privilegi menzionati siano concessi a medici anche altrove non è cogente, perché essi non sono esclusivi di questa categoria. Argomenti contro questa ipotesi in Manetti, Recensione.

${ }^{18}$ Ricciardetto, Lettre, 58-60, e Anonyme, CXXXII n. 430 e CXXXVII. Ricciardetto (pp. CXXXIVCXXXVII) si richiama anche a Debru, Démonstrations, equiparando le dimostrazioni spettacolari descritte da Galeno alle prove della gara.

${ }^{19}$ Cfr. Nutton, Meeting Place, 7-8. Le epigrafi che testimoniano le prove sono consultabili in Samama, Médecins, nn. 211, 212, 213 e 214, che sono in realtà elenchi di vincitori. Manca qualsiasi contesto che chiarisca la natura delle prove. La parola problema richiama naturalmente la letteratura, di origine peripatetica, dei Problemata, cioè le discussioni di singoli 'problemi', magari con la giustapposizione di soluzioni opposte. Anche se è attestato che a Roma, all'epoca di Galeno, a volte si sorteggiava un 'problema' che l'oratore doveva dimostrare di saper analizzare criticamente (Debru, Démonstrations), potrebbe trattarsi di una somiglianza lessicale ingannevole: Nutton parla infatti di "some form of diagnosis" che è qualcosa di completamente diverso. In ogni caso un testo come quello dell'Anonimo, che utilizza certamente la letteratura dei problemata, è troppo lungo e vario per essere una preparazione ad una prova del genere.

${ }^{20}$ Ebert, Brief.

${ }^{21}$ Manetti, Autografi e Anonymi.

${ }^{22}$ Del Corso, Politeia. 
o professionali e l'autore del papiro (che, secondo Del Corso, faceva parte del "gruppo di scrittura" menzionato sopra) l'ha fatto copiare per un interesse retorico. È necessario allora ipotizzare che esistessero manuali che raccoglievano testi di questo genere, inoltre la lettera non sembra particolarmente utile da questo punto di vista. L'ipotesi di Ricciardetto invece mira a spiegare proprio la motivazione della copia, ma si basa su fondamenta fragili. In conclusione mi pare che sia l'interpretazione del testo della lettera di Marco Antonio sia la sua presenza sul verso di P. Lond. inv. 137 non abbiano ancora trovato una soluzione soddisfacente e continuino a suscitare dubbi.

Occorre anche fare alcune precisazioni sullo scopo di questa scrittura autografa: Ricciardetto non condivide l'opinione di Manetti che si tratti del brogliaccio di un trattato (o meglio di un'opera introduttiva), rimasto incompiuto, e parla di composizione "per esercizio ${ }^{23}$, sia in funzione di una prova di concorso oppure semplicemente per sé. Il richiamo alle opere che Galeno afferma di avere composto per esercizio personale non basta a distinguere un esercizio da un brogliaccio. Infatti questi scritti di Galeno sono organizzati secondo le stesse convenzioni dei testi scritti per la pubblicazione ${ }^{24}$. Inoltre l'abilità compositiva dell'Anonimo è troppo sottovalutata: nonostante sia un compilatore, egli ha un suo progetto che è ben chiaro anche se interrotto. Partendo dalla definizione di 'affezione' e 'malattia' e passando dalla 'storia' delle dottrine eziologiche, egli comincia a esporre in forma sistematica le sue convinzioni sulla anatomia e fisiologia umana in una sezione che si intitola significativamente "Malattie" (iv 19), evidentemente come premessa della sua personale eziologia, che però non arriva a scrivere (almeno nel rotolo che ci è rimasto). È certamente influenzato dalla prassi dialettica di scuola e utilizza materiali del genere problemata ma offre molto di più.

Ci sono alcuni sicuri progressi nella lettura del testo dall'edizione di Manetti ${ }^{25}$ a quella di Ricciardetto ${ }^{26}$, anche in seguito alla recensione di David Leith che ha fatto alcune buone proposte: elenco qui sotto alcuni casi ${ }^{27}$.

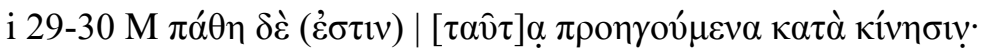

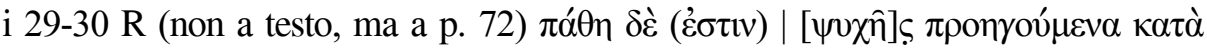
Kívฑб!v

\footnotetext{
${ }^{23}$ Ricciardetto, Anonyme, CXXXIV e n. 436.

${ }^{24}$ Gurd, है $\kappa \delta o \sigma l \varsigma$, Manetti, Exegesis, 1178.

${ }^{25}$ Manetti, Anonymi.

${ }^{26}$ Ricciardetto, Anonyme.

${ }^{27}$ Leith, Recensione. Id., Pores ha formulato anche altre proposte su un passo (vd. infra).
} 


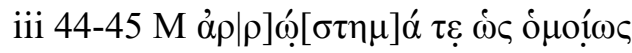

iii 44-45 R $\dot{\alpha} \rho \mid \rho] \omega[\sigma \tau i ́] \alpha \tau \varepsilon \dot{\omega} \varsigma \dot{o} \mu o^{\prime} \omega \varsigma$

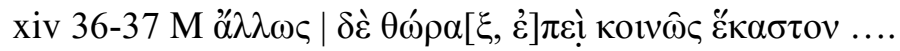

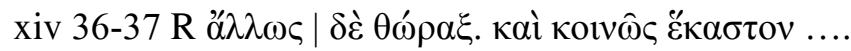

Nei tre casi precedenti si interpretano meglio le tracce in relazione agli spazi.

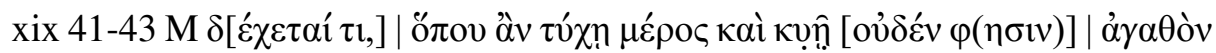

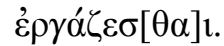

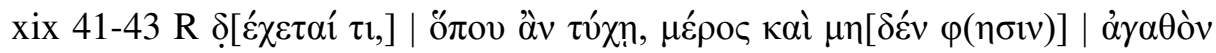

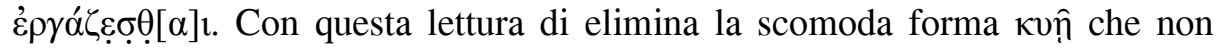
trovava adeguata spiegazione.

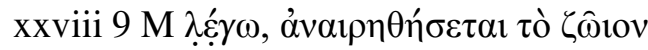

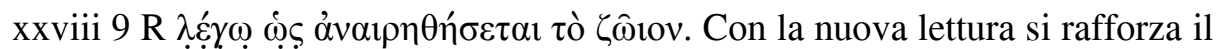
significato, dando maggiore valore autoriale all'uso da parte dell'Anonimo della prima persona singolare.

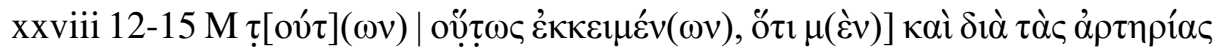

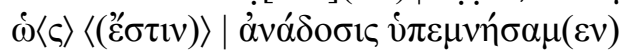

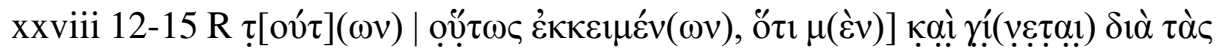

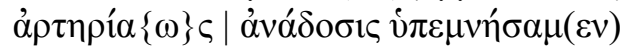

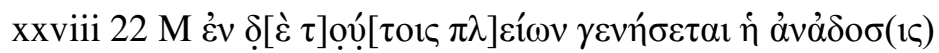

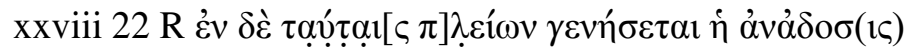

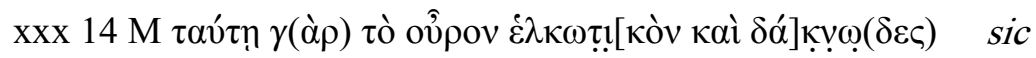

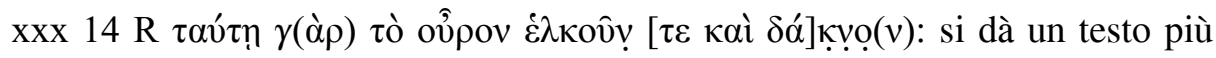
corretto e corrispondente allo spazio in lacuna.

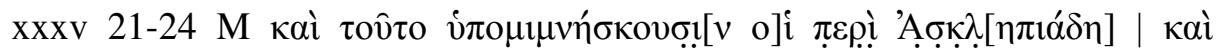

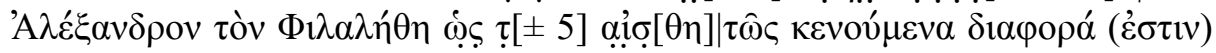

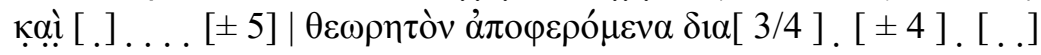




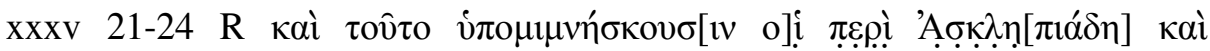

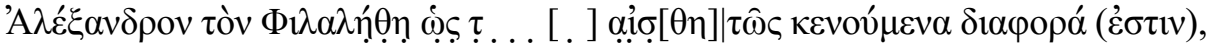

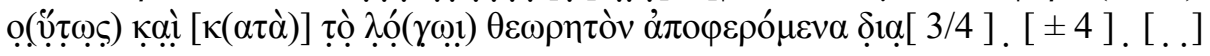

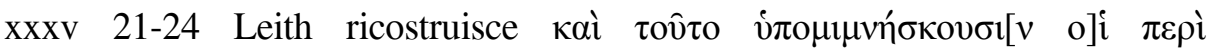

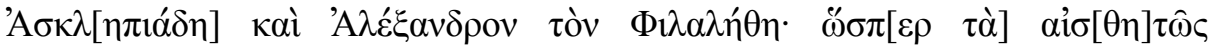

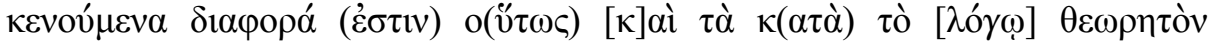

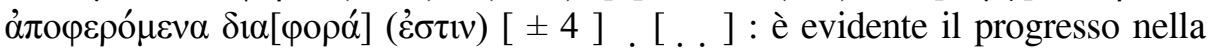
ricostruzione del testo.

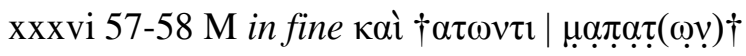

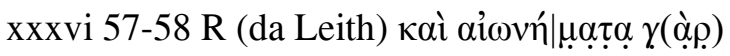

xxxix 10-14 M (R è sostanzialmente uguale, con differenze di dettaglio)

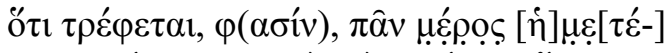

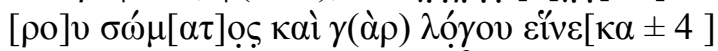

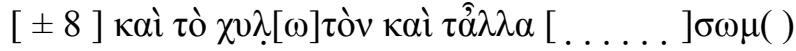

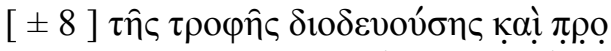

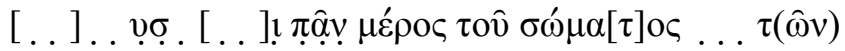

$[\lambda o ́] \gamma \omega \theta \varepsilon \omega \rho \eta ̣[\tau](\hat{\omega} v) \pi o ́ \rho \omega v$ óṿ $\tau(\omega v)$.

Xxxix 10-15 Leith $^{28}$

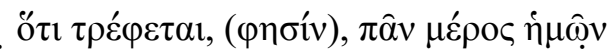

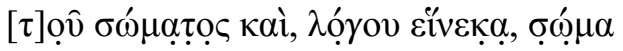

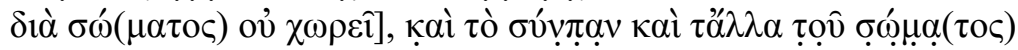

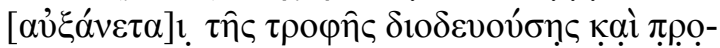

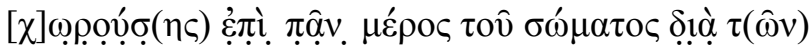

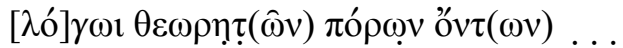

12 1. $\sigma u ́ \mu \pi \alpha v$

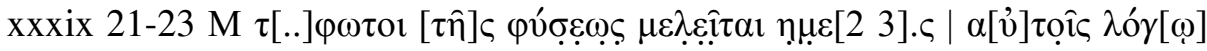

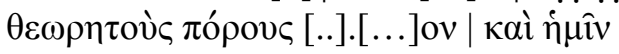

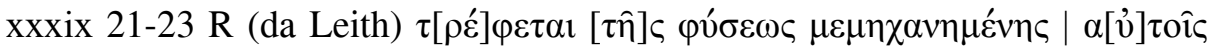

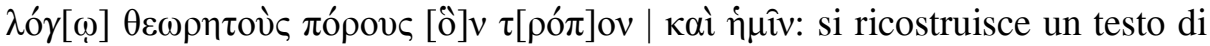
senso compiuto, che ripete il motivo della natura artefice, già comparso prima.

Vi sono però dei casi in cui le soluzioni di Ricciardetto (o di Leith) sono discutibili, per esempio:

\footnotetext{
${ }^{28}$ Leith, Pores, 174.
} 


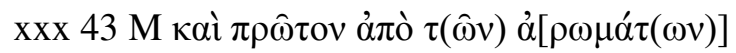

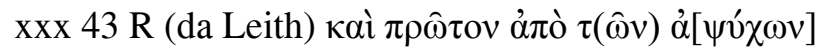

L'integrazione proposta da Leith è peggiore di quella di Diels, accolta da M. È vero che l'autore fa anche altrove riferimento agli esseri inanimati: egli usa il termine

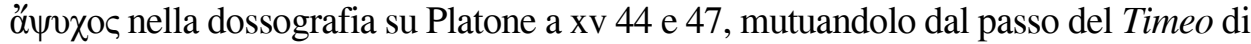
Platone che sta parafrasando ${ }^{29}$, e lo usa poi proprio all'inizio della sua argomentazione sull'esistenza di apophorai, a xxii 8-12, con una opposizione fra esseri animati $(\check{\varepsilon} \mu \psi v \chi \alpha)$ e inanimati ( $\alpha \chi \psi v \chi \alpha)$. La sua opinione è che ci sono maggiori emissioni dagli esseri animati che da quelli inanimati, a causa del calore e del movimento. Ma Leith non tiene conto dell'organizzazione e dell'ordinamento del discorso in questo punto, dove l'autore espone le prove dell'esistenza delle emissioni dal corpo in un ordine crescente in termini di scala naturae e arriva solo alla fine agli esseri inanimati, seguiti da piante, animali irrazionali e infine dall'uomo, come è chiaro dalla sequenza:

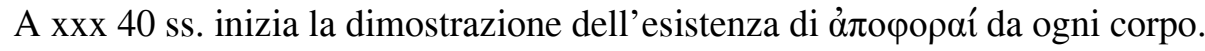

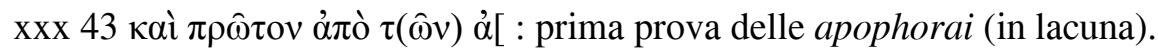

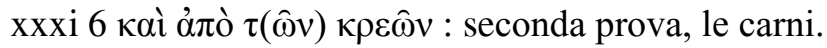

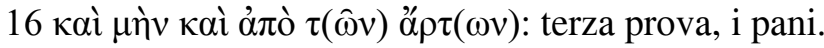

(lunga parentesi polemica sulla dimostrazione di ciò che è pesante o leggero).

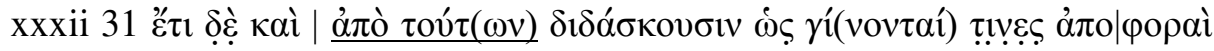

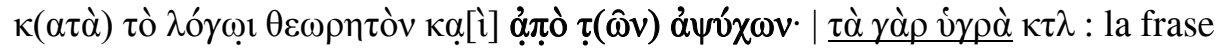
indica chiaramente un passaggio ad una nuova enumerazione di prove, in cui il termine $\alpha^{\prime} \psi v \chi 0 \varsigma$ si riferisce a ciò che segue, cioè i liquidi.

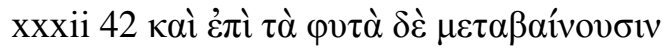

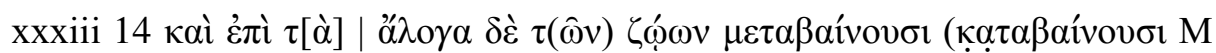
perperam)

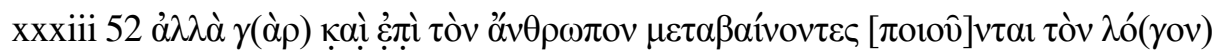

Un altro caso di interpretazione discutibile si trova a xxxv 26, dove l'aggiunta supra lineam leggibile sia come $\omega \varsigma \delta \eta$ sia come $\omega \varsigma \alpha \pi$ è stata messa a testo in modo differente in Manetti e in Ricciardetto:

$\mathrm{M} \dot{\omega} \varsigma \delta \hat{\eta}(\lambda \circ v)$

$\mathrm{R} \hat{\omega} \varsigma \dot{\alpha} \pi(\varepsilon \delta \varepsilon \dot{i} \xi \alpha \mu \varepsilon v)$, già proposta da Diels (ma con $\dot{\alpha} \pi\langle\varepsilon\rangle \delta \varepsilon^{\prime}[\xi] \alpha \mu \varepsilon \varphi$ in apparato)

\footnotetext{
${ }^{29}$ Cf. Manetti, Anonymi, 31 (apparato dei luoghi paralleli).
} 
Ritengo che la seconda soluzione sia sbagliata, ancora una volta sulla base dell'argomentazione dell'Anonimo: infatti la soluzione di Ricciardetto/Diels darebbe per dimostrata la tesi che le secrezioni visibili siano differenti e varie, mentre è proprio quello che l'autore intende fare nei righi successivi (27 ss.) che

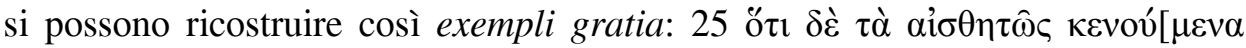

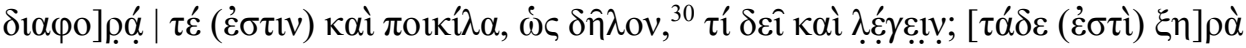

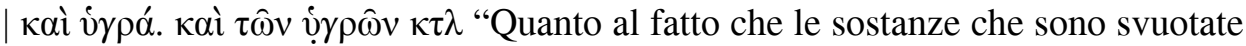
in modo visibile sono differenti e varie - come è evidente- cosa bisogna dire? Esse sono liquide e solide. E fra quelle liquide.....”. E si elencano tutti i vari tipi di secrezioni corporee (rr. 28 ss.), prima quelle liquide e poi (35 ss.) quelle solide.

Su alcuni punti del testo resta ancora molto lavoro da fare, ma si spera che altri decifrino meglio le tracce e riescano ad avanzare nuove proposte di lettura e integrazione. Comunque l'orizzonte, rispetto all'edizione di Hermann Diels del 1893, è completamente cambiato e promette nuovi sviluppi. Si auspicano nuovi contributi non solo da parte degli editori del testo, ma anche da parte degli studiosi che si occupano dei frammenti di Erofilo, Erasistrato, Asclepiade e Alessandro Filalete.

\section{Edizioni del testo}

Hermann Diels (cur.), Anonymi Londinensis ex Aristotelis Iatricis Menoniis et aliis medicis eclogae (Supplementum Aristotelicum, III.1), Berolini 1893.

Daniela Manetti (cur.), Anonymi Londiniensis de medicina, Berlin 2011.

Antonio Ricciardetto (cur.), L'Anonyme de Londres. Un papyrus médical grec du Ier siècle (Papyrologica Leodiensia 4), Liège 2014.

Antonio Ricciardetto (cur.), L'Anonyme de Londres. P.Lit.Lond. 165, Brit. Libr. inv. 137. Un papyrus médical grec du Ier siècle après J.-C., Paris 2016.

\section{Bibliografia}

Bastianini, Luogo = Guido Bastianini, Un luogo di ritrovamento fantasma, in: Atti del Convegno Internazionale di Egittologia e Papirologia, Siracusa 1-3 dicembre 1995 (Quaderni dell'Istituto Internazionale del Papiro 7), Siracusa 1996, 69-84.

Debru, Démonstrations $=$ Armelle Debru, Les démonstrations médicales à Rome au temps de Galien, in: Ph. van der Eijk / H. F. J. Horstmanshoff / P. H. Schrijvers (curr.), Ancient Medicine in Its Socio-Cultural Context, I, Amsterdam/Atlanta 2005, 69-80.

Del Corso, Politeia = Lucio Del Corso, L'Athenaion Politeia (P. Lit. Lond. 108) e la sua 'biblioteca': libri e mani nella chora egizia, in: D. Bianconi / L. Del Corso (curr.), Oltre la scrittura. Variazioni sul tema per Guglielmo Cavallo (Dossiers Byzantins 8), Paris 2008, 13-52.

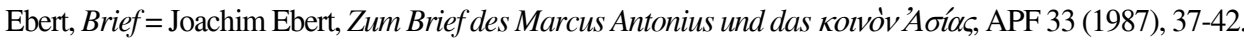

\footnotetext{
${ }^{30}$ Rispetto a Manetti, Anonymi, la virgola ha sostituito il punto in alto.
} 
Gurd, $\check{\varepsilon} \kappa \delta o \sigma \iota \varsigma=$ Sean A. Gurd, Galen on $\ddot{\varepsilon} \kappa \delta o \sigma \iota \varsigma$, in: T. Schmidt / P. Fleury (curr.), Perceptions of the Second Sophistic and Its Times, Toronto/Buffalo/London 2011, 169-84.

Kaltsas, Randnotizen $=$ Demokritos Kaltsas, Randnotizen zu einem Fund Griechischer Buchrollen (Aristoteles, Atheaion politeia, Herodas, u. a.), in: G. A. Xenis (cur.), Literature, Scholarship, Philosophy and History. Classical Studies in Memory of Ioannis Taifacos, Stuttgart 2015, 247-64.

Leith, Pores = David Leith, Pores and Void in Asclepiades' Physical Theory, "Phronesis" 57 (2012), 164-91.

Leith, Recensione = David Leith, recensione a Manetti, Anonymi, "Gnomon" 86 (2014), 592-5.

Manetti, Anonymi = Daniela Manetti (cur.), Anonymi Londiniensis de medicina, Berlin 2011.

Manetti, Authorial Presence $=$ Daniela Manetti, Levels of Authorial Presence in the Anonymus Londiniensis (P.Br. Libr.inv. 137), "Trends in Classics" 5 (2013), 159-78.

Manetti, Autografi = Daniela Manetti, Autografi e incompiuti: il caso dell'Anonimo Londinese P. Lit. Lond. 165, ZPE 100 (1994), 47-58.

Manetti, Doxography = Daniela Manetti, 'Aristotle' and the Role of Doxography in the Anonymus Londiniensis (PBrLibrinv. 137), in: Ph. van der Eijk (cur.), Ancient Histories of Medicine. Essays in Medical Doxography and Historiography in Classical Antiquity (SAM 20), Leiden/Boston 1999, 95-141.

Manetti, Exegesis = Daniela Manetti, Medicine and Exegesis, in: F. Montanari / S. Matthaios / A. Rengakos (curr.), Brill's Companion to Ancient Greek Scholarship, Leiden/Boston 2015, 1126-215.

Manetti, Recensione $=$ Daniela Manetti, recensione a Ricciardetto, L'Anonyme de Londres, Liège 2014, "Lettre d'information" n.s. 12 (2015), 80-5.

Messeri, PLitLond 131 = Gabriella Messeri, PLitLond 131: Isocrates, 'De pace', in: A. Carlini / D. Manetti (curr.), Studi sulla tradizione del testo di Isocrate, Firenze 2003, 21-54.

Nissen, Asclépios = C. Nissen, Entre Asclépios et Hippocrate. Étude des cultes guérisseurs et des médecins en Carie (Kernos, suppl. 22), Liège 2009.

Nutton, Meeting Place = Vivian Nutton, The Medical Meeting Place, in: Ph. van der Eijk / H. F. J. Horstmanshoff / P. H. Schrijvers (curr.), Ancient Medicine in Its Socio-Cultural Context, I, Amsterdam/Atlanta 2005, 3-25.

Ricciardetto, Abréviations = Antonio Ricciardetto, Abréviations et signes dans l'Anonyme de Londres, in: N. Carlig / G. Lescuyer / A. Motte / N. Sojic (edd.), Signes dans les textes. Recherches sur les continuités et les ruptures des pratiques scribales en Égypte pharaonique, gréco-romaine et byzantine. Actes du colloque international, Université de Liège, 2-4 juin 2016 (Papyrologica Leodiensia), Liège, in pubblicazione.

Ricciardetto, Comparaison $=$ Antonio Ricciardetto, Comparaison entre le systeme d'abréviations de l'Anonyme de Londres et ceux de la Constitution d'Athènes et des autre textes littéraires du Brit. Libr. inv. 131, in: S. Torallas Tovar / A. Nodar (curr.), Proceedings of the 28th International Congress of Papyrology, Barcelona 2016. Barcelona, in pubblicazione.

Ricciardetto, Lettre = Antonio Ricciardetto, La lettre de Marc Antoine (SB I 4224) écrite au verso de l'Anonyme de Londres (P.Brit. Libr. inv. 137 = MP3 2339), APF 58 (2012), 43-60.

Ricciardetto, Recensione $=$ Antonio Ricciardetto, recensione a Manetti, Anonymi, "Aestimatio" 10 (2013), 79-98.

Ricciardetto, Spazio scritto = Antonio Ricciardetto, Spazio scritto e spazio non scritto nelle dossografie mediche su papiro, in: N. Pellé (cur.), Spazio scritto e spazio non scritto nel libro papiraceo. Esperienze a confronto, Atti della Seconda Tavola Rotonda del Centro di Studi Papirologici dell'Università del Salento, 9 ottobre 2014, Lecce 2016, 183-224.

Samama, Médecins = Evelyne Samama, Les médecins dans le monde grec, Genève 2003. 
